\title{
The effect of early menarche on the sexual behaviors of Korean female adolescents
}

\author{
Ji In Cheong, MD', \\ Chang Hun Lee, MD', \\ Jae Hong Park, MD, PhD', \\ Byeong Jin $\mathrm{Ye}, \mathrm{MD}, \mathrm{PhD}^{3}$, \\ Kyoung Ah Kwon, MD', \\ Young Seok Lee, MD, PhD', \\ Jae Ho Yoo, MD'

\section{Departments of ${ }^{1}$ Pediatrics, ${ }^{2}$ Psychiatry, and ${ }^{3}$ Occupational and Environmental Medicine, Dong-A University College of Medicine, Busan, Korea}

\begin{abstract}
Purpose: This study examined the relationships between early menarche and sexual behaviors among Korean female adolescents.

Methods: We analyzed data from the eighth Korea Youth Risk Behavior Web-based survey that was conducted on female high school students in grades 10-12. The survey included 17,867 students, and 974 students were assigned to the early menarche group because they had experienced menarche when they were in grade four or below, and 16,893 students were assigned to the normal menarche group because they had experienced menarche during or after grade five. The characteristics of the sexual behaviors in the early menarche and normal menarche group were analyzed.

Results: The early menarche group was at a higher risk of intersexual kissing or petting (odds ratio [OR], 1.54; 95\% confidence interval [Cl], 1.28-1.87), intersexual intercourse $(\mathrm{OR}, 2.35 ; 95 \% \mathrm{Cl}, 1.65-3.36)$, homosexual kissing or petting (OR, 3.53; $95 \% \mathrm{Cl}, 2.22-5.59)$, homosexual intercourse $(\mathrm{OR}, 7.70 ; 95 \% \mathrm{Cl}, 4.04-14.66)$, being the victim (OR, 2.89; $95 \% \mathrm{Cl}, 1.98-4.22)$ or the assailant (OR, 13.55; 95\% Cl, 6.61-27.78) of sexual assaults, intercourse without any contraception (OR, 1.92; $95 \% \mathrm{Cl}, 1.06-3.46)$, and pregnancy $(\mathrm{OR}, 5.72 ; 95 \% \mathrm{Cl}, 2.31-14.15)$ than the normal menarche group.

Conclusion: Early menarche is associated with risky sexual behaviors among adolescent females; therefore, developing comprehensive sexual health education programs and tools for early intervention are required for children who are expected to experience early menarche.
\end{abstract}

Keywords: Sexual behavior, Menarche, Precocious puberty

\section{Introduction}

Unlike American and European women whose menarcheal ages gradually declined during the 19th century and were maintained at a mean age of 12.5 years during the 20th century ${ }^{1}$, the age at menarche for Korean women has shown a tendency to reduce rapidly over the last 50 years. Therefore, the average age at menarche for women who were born between 1990 and 1994 had declined to $12.60 \pm 1.14$ years $^{2}$. This relatively rapid change in the age at menarche requires more attention with regard to the effects of early menarche on women's health in Korea. Early menarche increases the incidence of obesity, metabolic syndrome ${ }^{2)}$, and type 2 diabetes mellitus ${ }^{3)}$, and it is associated with the occurrence of breast cancer ${ }^{4)}$ and polycystic ovarian syndrome ${ }^{5)}$. Furthermore, it is associated with early skeletal maturation, which results in shorter final adult heights ${ }^{6}$. Early menarche not only causes health-related problems, but it also increases the risks of psychological problems, including depression, psychosomatic symptoms, delinquency ${ }^{7}$.

This study aimed to analyze the effects of early menarche on the sexual behaviors of Korean adolescents. We used data from the Korea Youth Risk Behavior Web-based survey ${ }^{8}$, which was jointly conducted by the Ministry of Education, Ministry of Health and Welfare, and the Korea
Address for correspondence:

Jae Ho Yoo, MD

Department of Pediatrics, Dong-A

University Hospital, Dong-A

University College of Medicine, 26

Daesingongwon-ro, Seo-gu, Busan

49201, Korea

Tel: +82-51-240-2883

Fax: +82-51-242-2765

E-mail: pedendo@dau.ac.kr 
Centers for Disease Control and Prevention, and we compared a group of females who had undergone early menarche with a group of females who had undergone menarche at a normal age, and the differences in the sexual behaviors between the groups were analyzed.

\section{Materials and methods}

\section{Subjects}

This study used data from the eighth Korea Youth Risk Behavior Web-based survey conducted in 2012. The Ministry of Education, the Ministry of Health and Welfare, and the Korea Centers for Disease Control and Prevention have conducted this self-administered Web-based survey on middle and high school students in grades 7-12 every year since 2005 . The 2012 survey was conducted on 76,980 students from 800 schools, which included 400 middle schools and 400 high schools. A total of 74,186 students from 797 schools participated in this survey. To minimize the standard deviations, regions were categorized as large cities, medium-to-small cities, and counties, and they were reclassified to account for geographical accessibility, the numbers of schools, the sizes of the populations, and the living environments. The schools were divided into middle schools, general high schools, and specialized high schools, and a number of sample schools were selected based on the city, the province, the size of the city, and whether the schools were boys', girls', or coeducational schools. After the sample schools had been selected, one class from each grade was randomly selected and every student in that class was surveyed, with the exception of students who were on long-term absences, handicapped children, and students with dyslexia.

Female high school students who were in grades 10-12 were included in this study, and the data were analyzed after the students had been divided into an early menarche group and a normal menarche group. The early menarche group was defined as female students who underwent menarche when they were in grade four or below (sgrade 4 ), and the normal menarche group was defined as female students who had undergone menarche during or after grade five ( $\geq$ grade 5 ). Female students who had not undergone menarche at the time of the survey were excluded from the study.

\section{Methods}

The Korea Youth Risk Behavior Web-based survey was conducted during computer classes when internet connections were available, and one computer was randomly assigned to a student who answered the questionnaire anonymously.

The students were divided into the early menarche group and the normal menarche group, which was the control group, using the survey's statistics, and the differences between the groups were analyzed with respect to intersexual and homosexual kissing, petting, intercourse, and being the victims or assailants of sexual assaults. In addition, intercourse without using any contraceptive devices and histories of pregnancy were investigated among the students who had experienced sexual intercourse.

Factors that were considered to have impacts on these sexual behaviors, including the age at menarche, the parents' educational levels, the living status, the academic performance, and the household income, were examined to determine their relationships with the students' sexual behaviors.

The parents' educational levels were categorized as "less than high school", "beyond high school", and "beyond college", and the students were asked to select one answer. If they did not respond to the question, this was treated as a missing value. The student's living status was categorized into "yes" or "no", which indicated whether or not the student lived with both parents. The student's academic performance was categorized as "poor", "average", and "well", and the household income was classified as "low", "medium", and "high".

\section{Statistical analysis}

The data from this study were stratified because of the complex nature of the survey's design and the data. Therefore, the survey's weights and design effects were taken into account during the statistical analysis to confirm the effects of the factors. The characteristics of the subjects were compared using the chisquare test. Multivariate regression analysis was performed to analyze the risk factors based on the differences in the students' sexual behaviors. All of the statistical analyses were performed using IBM SPSS Statistics ver. 20.0 (IBM Co., Armonk, NY, USA), and a $P$-value of $<0.05$ was considered statistically significant.

\section{Results}

\section{Demographic characteristics of the subjects}

A total of 17,867 female high school students had undergone menarche, and, of these, 974 students (5.45\%) were assigned to the early menarche group, and 16,893 students (94.55\%) were assigned to the normal menarche group. Compared with the normal menarche group, more students in the early menarche group responded that their paternal educational levels were "less than high school" and "beyond high school" ( $P=0.008)$, and 2,083 students did not respond to the question about the paternal educational level. More students in the early menarche group responded that their maternal educational levels were "less than high school" and "beyond high school" compared with the normal menarche group $(P=0.011)$, and 1,652 students did not respond to the question about the maternal educational level. More students in the early menarche group did not live with both parents compared with the normal menarche group $(P<0.001)$. There was no significant difference between the groups regarding the students' academic performances. Compared with the normal 
menarche group, more students in the early menarche group answered "low" to the household income question $(P<0.001)$ (Table 1).

\section{Sexual behaviors arising from early menarche}

After correcting for the confounding factors that included the parents' educational levels, the living status, the academic performance, and the household income, significant differences were apparent between the groups with respect to the students sexual behaviors based on the menarcheal age.

The early menarche group had more experience in intersexual kissing or petting than the normal menarche group (odds ratio $[\mathrm{OR}], 1.54 ; 95 \%$ confidence interval [CI], 1.28-1.87). The early menarche group had more experience in intersexual intercourse than the normal menarche group (OR, 2.35; 95\% CI, 1.65-3.36). Compared with the normal menarche group, the early menarche group had more experience in homosexual kissing or petting $(\mathrm{OR}$, 3.53 ; 95\% CI, 2.22-5.59) and in homosexual intercourse (OR, 7.70; 95\% CI, 4.04-14.66). The students in the early menarche group had more experience in being the victims of sexual assaults (OR, 2.89; 95\% CI, 1.98-4.22) and in being the assailants of sexual assaults (OR, 13.55; 95\% CI, 6.61-27.78) than the normal menarche group. The early menarche group had more experience of intercourse without using any contraception (OR, 1.92; 95\% CI, 1.06-3.46) and a higher frequency of pregnancy (OR, 5.72; $95 \% \mathrm{CI}, 2.31-14.15)$ than the normal menarche group (Table 2).

\section{Discussion}

This study focused on the relationship between early menarche and sexual behaviors, and used a multivariate logistic regression model to determine whether the relationship between early menarche and sexual behaviors was significant, after correcting for social background factors that may affect sexual behaviors. In this study, the early menarche group tended to not live with their parents and have parents with relatively lower education levels and economic statuses than the normal menarche group. The results of similar previous studies differ. In a study conducted on 1,315 participants in England, those who had experienced menarche before the age of 12 years tended to have lower economic status than those who had experienced menarche after the age of 13 years (OR 1.32; 95\% CI, 1.03-1.71) . Another study on the relationship between early menarche and social and environmental factors in 3,388 participants demonstrated no statistically significant correlations between the absence of parents or low economic status and early menarche ${ }^{10)}$. In a study conducted on 1,091 African American female adolescents and 986 white female adolescents, no significant correlations were found between early menarche and parents' education level, although the risk of early menarche increased with higher household income in African American adolescents (OR, 2.15; 95\% CI, 1.27-3.63) and decreased with higher household income in white adolescents (OR, 0.37; 95\% CI, 0.18 $0.80)^{11)}$. Considering the results of these studies and our study,

Table 1. Characteristics of the subgroups defined by age at menarche

\begin{tabular}{|c|c|c|c|}
\hline Characteristic & Early menarche group & Normal menarche group & $P$-value \\
\hline \multicolumn{4}{|l|}{ Paternal educational level } \\
\hline Less than high school & $71(8.6)$ & $897(6.0)$ & \\
\hline Beyond high school & $366(44.1)$ & $6,602(44.1)$ & 0.008 \\
\hline College/university degree & $392(47.3)$ & $7,456(49.8)$ & \\
\hline Total & $829(100.0)$ & $14,955(100.0)$ & \\
\hline \multicolumn{4}{|l|}{ Maternal educational level } \\
\hline Less than high school & $65(7.5)$ & $915(6.0)$ & \\
\hline Beyond high school & $509(58.7)$ & $8,851(57.7)$ & 0.011 \\
\hline College/university degree & $293(33.8)$ & $5,582(36.4)$ & \\
\hline Total & $867(100.0)$ & $15,348(100.0)$ & \\
\hline \multicolumn{4}{|l|}{ Living with both parents } \\
\hline Yes & $743(76.3)$ & $13,796(81.7)$ & \\
\hline No & $231(23.7)$ & 3,097 (18.3) & $<0.001$ \\
\hline Total & $974(100.0)$ & $16,893(100.0)$ & \\
\hline \multicolumn{4}{|l|}{ Academic performance } \\
\hline Poor & $401(41.2)$ & $6,577(38.9)$ & \\
\hline Average & $264(27.1)$ & $5,094(30.2)$ & 0.081 \\
\hline Well & $309(31.7)$ & $5,222(30.9)$ & \\
\hline Total & $974(100.0)$ & $16,893(100.0)$ & \\
\hline \multicolumn{4}{|l|}{ Household income } \\
\hline Low & $331(34.0)$ & $4,764(28.2)$ & \\
\hline Middle & $412(42.3)$ & $8,380(49.6)$ & $<0.001$ \\
\hline High & $231(23.7)$ & $3,749(22.2)$ & \\
\hline Total & $974(100.0)$ & $16,893(100.0)$ & \\
\hline
\end{tabular}

Values are presented as number (\%). 
additional studies on socioeconomic factors that influence the age at menarche may be required.

This study's findings showed that the early menarche group had higher levels of intersexual and homosexual experiences compared with the normal menarche group, and this concurs with the results from earlier studies. In a study of 1,420 adolescents, females who had undergone menarche early had experienced sexual intercourse at earlier ages (OR, 2.1; 95\% CI, 1.0-4.3), and, when they became adults, they were at a higher risk of having more than 10 sexual partners (OR, 10.0; 95\% CI, $1.2-85.9)^{12)}$. In another study of 8,232 women, the group that had undergone menarche at $14-15$ years of age (OR, 0.31 ; $95 \%$ CI, 0.26-0.36) and the group that had undergone menarche after 16 years of age (OR, 0.04; 95\% CI, 0.02-0.05) were at lower risks of having sexual intercourse before 15 years of age compared with the group that had undergone menarche before the age of 14 years ${ }^{13)}$. The findings from a cohort study of 497 women showed that early pregnancies and sexually transmitted infections were strongly associated with the age at menarche, and that these results were upheld after adjusting for the confounding factors that included family background, family functionality, and individual factors. In addition, the risks of pregnancy during adolescence and sexually transmitted infections increased as the age at menarche declined ${ }^{14)}$. In this study, the risks of having intercourse without using any contraception and pregnancy were 1.92 folds and 5.72 folds higher, respectively, in the early menarche group compared with the normal menarche group. Hence, the early menarche group was at an increased risk of pregnancy during adolescence. Therefore, actively implemented early sexual health education programs are necessary to prevent early pregnancies.

The risks of homosexual experiences and intercourse were high in the early menarche group in this study. It has been reported that more than $80 \%$ of the women who were sexually attracted to the same sex during their teenage years became sexually attracted to the opposite $\operatorname{sex}^{15)}$. In addition, it has been reported that the age at menarche for lesbian or bisexual women does not differ from that of heterosexual women ${ }^{16)}$. Therefore, the high risks of homosexual experiences and intercourse associated with the early menarche group do not indicate that these students have high probabilities of becoming lesbian or bisexual women. However, since children at young ages tend to maintain close relationships with friends of the same sex, it can be assumed that their responses to sex hormones may feel like sexual attraction, and that this may develop into homosexual experiences. Therefore, we should be careful not to stigmatize these children by misinterpreting their behaviors as homosexuality.

Table 2. The effect of early menarche on the sexual behaviors from the Korea Youth Risk Behavior Web-based survey (2012)

\begin{tabular}{|c|c|c|c|}
\hline Variable & Early menarche & Normal menarche & $\mathrm{OR}^{\mathrm{a})}(95 \% \mathrm{Cl})$ \\
\hline \multicolumn{4}{|c|}{ Intersexual kissing or petting } \\
\hline Yes & 254/974 (26.1) & 2,323/16,090 (14.4) & $1.54(1.28-1.87)$ \\
\hline No & 720/974 (73.9) & $13,767 / 16,090(85.6)$ & \\
\hline \multicolumn{4}{|c|}{ Intersexual intercourse } \\
\hline Yes & 67/974 (6.9) & $522 / 16,893(3.1)$ & $2.35(1.65-3.36)$ \\
\hline No & 907/974 (93.1) & $16,371 / 16,893(96.9)$ & \\
\hline \multicolumn{4}{|c|}{ Homosexual kissing or petting } \\
\hline Yes & $34 / 974(3.5)$ & $167 / 16,893(1.0)$ & $3.53(2.22-5.59)$ \\
\hline No & $940 / 974(96.5)$ & 16,726/16,893 (99.0) & \\
\hline \multicolumn{4}{|c|}{ Homosexual intercourse } \\
\hline Yes & 25/974 (2.6) & $55 / 16,893(0.3)$ & $7.70(4.04-14.66)$ \\
\hline No & 949/974 (97.4) & $16,838 / 16,893(99.7)$ & \\
\hline \multicolumn{4}{|c|}{ Victims of sexual assault } \\
\hline Yes & $54 / 974(5.5)$ & $302 / 16,893(1.8)$ & $2.89(1.98-4.22)$ \\
\hline No & $920 / 974(94.5)$ & $16,591 / 16,893(98.2)$ & \\
\hline \multicolumn{4}{|c|}{ Assailants of sexual assault } \\
\hline Yes & 23/974 (2.4) & $40 / 16,893(0.2)$ & $13.55(6.61-27.78)$ \\
\hline No & $951 / 974(97.6)$ & $16,853 / 16,893(99.8)$ & \\
\hline \multicolumn{4}{|c|}{ Intercourse without any contraception } \\
\hline Yes & $46 / 73(63.0)$ & 294/556 (52.9) & $1.92(1.06-3.46)$ \\
\hline No & 27/73 (37.0) & $262 / 556(47.1)$ & \\
\hline \multicolumn{4}{|c|}{ History of pregnancy } \\
\hline Yes & 20/72 (27.8) & $34 / 554(6.1)$ & $5.72(2.31-14.15)$ \\
\hline No & $52 / 72(72.2)$ & $520 / 554(93.9)$ & \\
\hline
\end{tabular}

Values are presented as number (\%)

$\mathrm{OR}$, odds ratio; $\mathrm{Cl}$, confidence interval.

${ }^{a}$ Odds ratio (adjusted for sociodemographic variables of paternal educational level, maternal educational level, living with both parents, academic performance and household income) for early menarche group for event compared with normal menarche group. 
The findings from this study demonstrated that early menarche was associated with a higher risk of being the victim of a sexual assault. The findings from a study of 3,288 women aged 18 years or older showed that menarche before the age of 11 years was strongly associated with sexual abuse (OR, 2.19; $95 \% \mathrm{CI}, 1.54-3.12)^{10)}$, and the findings from a study of 323 African-American and multiethnic Caucasian women demonstrated that menarche that occurred at an earlier age was associated with a higher likelihood of childhood sexual abuse ${ }^{17)}$. Since the early menarche group was more likely to have been exposed to sexual abuse, measures to prevent sexual abuse are required for adolescents who have undergone menarche early and for those who have experienced precocious puberty and are expected to undergo menarche early. In addition, the risk of being an assailant of a sexual assault was 13.55 folds higher in the early menarche group than in the normal menarche group in this study. Findings from a cross-sectional Web-based survey conducted on 1,086 adolescents in southeastern Michigan revealed that nearly half of the respondents (45.8\%) had been sexually assaulted by their friends ${ }^{18}$. This could be viewed as sexual curiosity and the inappropriate resolution of sexual impulses by targeting those who are close. We believe that being younger at the beginning of puberty was the reason underlying the higher risk of individuals being assailants of sexual assaults in the early menarche group. However, the question in the self-administered questionnaire used in this study was "Have you ever experienced being an assailant of a sexual assault", which does not allow for information to be provided about the methods used in or the degree of the sexual assaults, or the existence of genuine victims. One report has suggested that when more specific questions are included in surveys that ask respondents about their sexual assault experiences, for example, the type and degree of the sexual behavior, the place, and the subject, the prevalence of sexual assault assailants is higher than when a survey with a single question is conducted ${ }^{18)}$. Therefore, more specific questions should be included in surveys used in future studies.

This study was limited because the original data contained information about the grades during which the students underwent menarche, so the exact menarcheal ages had not to be estimated. This study defined early menarche as students who had undergone menarche when they were in grade four or below ( $\leq$ grade 4 ), and it is estimated that the secondary sexual characteristics had developed before grade two in this group. Given that the primary school entrance age is 6 years in Korea, the students in the early menarche group were thought to have been younger than 8 years of age at the onset of puberty, and they were thought to have been younger than 10 years of age when they experienced menarche. The students in the early menarche group in this study accounted for $5.4 \%$ of the total number of study subjects. Therefore, the results of this study can be viewed as being significant, because they were derived from a study on sexual behaviors in which about $5 \%$ of all Korean adolescents experienced the early onset of puberty. Another limitation of this study is that the analysis of the data was based upon the participants responses to a self-administered Webbased survey, which might have led to recollection errors or selection errors. In future studies, the accuracy of the data should to be improved by including a variety of information sources, for example, family members and cohabitants as well as the adolescent females. In addition, we did not account for the social or psychological factors that could influence the respondents' sexual behaviors, except for the parents' educational levels, whether the students lived with both parents, the participants' academic performances, and the factors associated with household income. Some studies have reported that early menarche is associated with psychological instability, including depression, anxiety, and aggression, and that this causes high rates of impulsive behaviors ${ }^{19,20)}$. A comprehensive evaluation of the factors, for example, the relationships between the adolescents and their parents or peers, and the adolescents' psychological statuses, is required in future studies.

In conclusion, early menarche is strongly associated with early sexual intercourse and teenage pregnancies, and the early menarche group in this study was considered a highrisk group for sexual assaults. Therefore, the development of a comprehensive and easily understood sexual health education program is needed for those children who have undergone early menarche and for those who have experienced precocious puberty and are expected to undergo early menarche. In addition, the sexual health education program must be promoted among caregivers, including parents and teachers, to ensure their commitment to the program.

\section{Conflict of interest}

No potential conflict of interest relevant to this article was reported.

\section{References}

1. Al-Sahab B, Adair L, Hamadeh MJ, Ardern CI, Tamim H. Impact of breastfeeding duration on age at menarche. Am J Epidemiol 2011;173:971-7.

2. Ahn JH, Lim SW, Song BS, Seo J, Lee JA, Kim DH, et al. Age at menarche in the Korean female: secular trends and relationship to adulthood body mass index. Ann Pediatr Endocrinol Metab 2013;18:60-4.

3. Lim JS, Lee HS, Kim EY, Yi KH, Hwang IS. Early menarche increases the risk of Type 2 diabetes in young and middleaged Korean women. Diabet Med 2015;32:521-5.

4. Shin A, Song YM, Yoo KY, Sung J. Menstrual factors and cancer risk among Korean women. Int J Epidemiol 2011;40:1261-8.

5. Magiakou MA, Manousaki D, Papadaki M, Hadjidakis D, Levidou G, Vakaki M, et al. The efficacy and safety of gonadotropin-releasing hormone analog treatment in childhood and adolescence: a single center, long-term follow-up study. J Clin Endocrinol Metab 2010;95:109-17. 
6. Kwon EB, Lee SJ, Cha M, Kim SY. Changes in the predicted adult height after gonadotropin-releasing hormone agonist treatment in girls with idiopathic true precocious puberty. Ann Pediatr Endocrinol Metab 2012;17:160-8.

7. Mendle J, Turkheimer E, Emery RE. Detrimental psychological outcomes associated with early pubertal timing in adolescent girls. Dev Rev 2007;27:151-71.

8. Korea Centers for Disease Control and Prevention. The eighth Korea youth risk behavior Web-based survey, 2012 [Internet]. Seoul: Ministry of Education, Science and Technology, Ministry of Health and Welfare, Korea Centers for Disease Control and Prevention; 2012 [cited 2014 Nov 7]. Available from: http://yhs.cdc.go.kr.

9. Downing J, Bellis MA. Early pubertal onset and its relationship with sexual risk taking, substance use and anti-social behaviour: a preliminary cross-sectional study. BMC Public Health 2009;9:446.

10. Henrichs KL, McCauley HL, Miller E, Styne DM, Saito N, Breslau J. Early menarche and childhood adversities in a nationally representative sample. Int J Pediatr Endocrinol 2014;2014:14.

11. Braithwaite D, Moore DH, Lustig RH, Epel ES, Ong KK, Rehkopf DH, et al. Socioeconomic status in relation to early menarche among black and white girls. Cancer Causes Control 2009;20:713-20.

12. Copeland W, Shanahan L, Miller S, Costello EJ, Angold A, Maughan B. Do the negative effects of early pubertal timing on adolescent girls continue into young adulthood? Am J
Psychiatry 2010;167:1218-25.

13. Glynn JR, Kayuni N, Floyd S, Banda E, Francis-Chizororo M, Tanton C, et al. Age at menarche, schooling, and sexual debut in northern Malawi. PLoS One 2010;5:e15334.

14. Boden JM, Fergusson DM, Horwood LJ. Age of menarche and psychosocial outcomes in a New Zealand birth cohort. J Am Acad Child Adolesc Psychiatry 201 1;50:132-40.e5.

15. Tulloch T, Kaufman M. Adolescent sexuality. Pediatr Rev 2013;34:29-37.

16. Bogaert AF, Friesen C, Klentrou P. Age of puberty and sexual orientation in a national probability sample. Arch Sex Behav 2002;31:73-81.

17. Zabin LS, Emerson MR, Rowland DL. Childhood sexual abuse and early menarche: the direction of their relationship and its implications. J Adolesc Health 2005;36:393400 .

18. Young AM, Grey M, Boyd CJ. Adolescents' experiences of sexual assault by peers: prevalence and nature of victimization occurring within and outside of school. J Youth Adolesc 2009;38:1072-83.

19. Mrug S, Elliott MN, Davies S, Tortolero SR, Cuccaro P, Schuster MA. Early puberty, negative peer influence, and problem behaviors in adolescent girls. Pediatrics 2014;133:7-14.

20. Rodgers JL, Van Hulle C, D'Onofrio B, Rathouz P, Beasley W, Johnson A, et al. Behavior problems and timing of menarche: a developmental longitudinal biometrical analysis using the NLSY-Children data. Behav Genet 2015;45:51-70. 